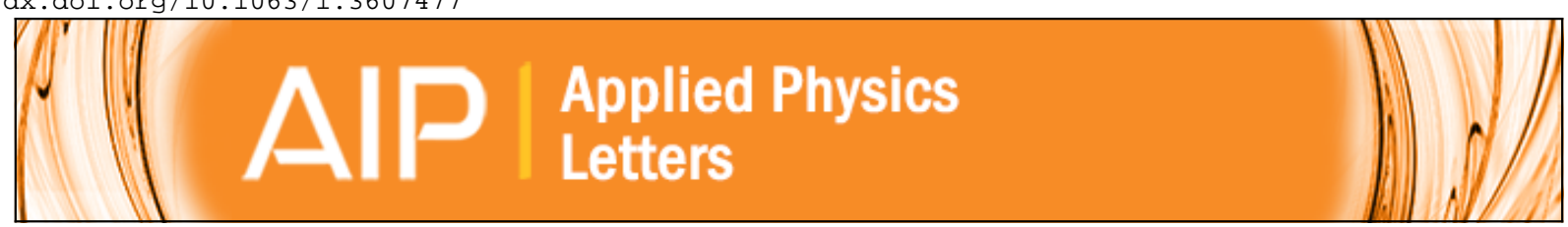

Heat conduction across a solid-solid interface: Understanding nanoscale interfacial effects on thermal resistance

Ganesh Balasubramanian and Ishwar K. Puri

Citation: Applied Physics Letters 99, 013116 (2011); doi: 10.1063/1.3607477

View online: http://dx.doi.org/10.1063/1.3607477

View Table of Contents: http://scitation.aip.org/content/aip/journal/apl/99/1?ver=pdfcov

Published by the AIP Publishing

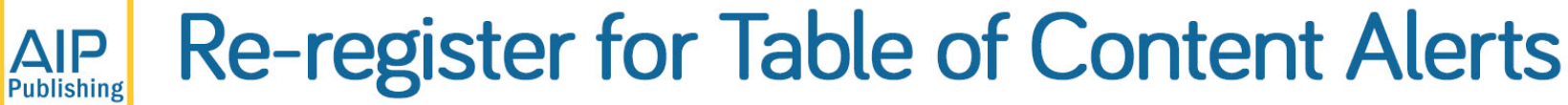




\title{
Heat conduction across a solid-solid interface: Understanding nanoscale interfacial effects on thermal resistance
}

\author{
Ganesh Balasubramanian and Ishwar K. Puria \\ Department of Engineering Science and Mechanics, Virginia Tech, Blacksburg, Virginia 24061, USA
}

(Received 9 April 2011; accepted 6 June 2011; published online 8 July 2011)

\begin{abstract}
Phonons scatter and travel ballistically in systems smaller than the phonon mean free path. At larger lengths, the transport is instead predominantly diffusive. We employ molecular dynamics simulations to describe the length dependence of the thermal conductivity. The simulations show that the interfacial thermal resistance $R_{k}$ for a $\mathrm{Si}-\mathrm{Ge}$ superlattice is inversely proportional to its length, but reaches a constant value as the system dimension becomes larger than the phonon mean free path. This nanoscale effect is incorporated into an accurate continuum model by treating the interface as a distinct material with an effective thermal resistance equal to $R_{k}$. (C) 2011 American Institute of Physics. [doi:10.1063/1.3607477]
\end{abstract}

The heat conduction mechanism in solids varies across lengthscales. ${ }^{1,2}$ Phonons, which enable energy transfer through lattice vibrations, are the predominant energy carriers in dielectrics and semiconductors. ${ }^{1}$ The ballistic propagation of phonons along dimensions smaller than the phonon mean free path causes them to scatter at the system boundaries. ${ }^{2,3}$ For larger $(>50 \mathrm{~nm}$ ) length scales, internal phonon scattering within the material produces diffusive heat transfer. ${ }^{4}$ This scattering, which can depend upon the direction of heat flux, ${ }^{5}$ is enhanced as the phonon wavelength becomes comparable to the length scales of impurities and interfaces. ${ }^{6,7}$

An interface between dissimilar materials, even without defects on the contact surfaces, creates an impedance to thermal transport that depends upon the differences in the densities and phonon propagation speeds for the two materials. The impedance induces an interfacial thermal (or Kapitza) resistance ${ }^{8-11} R_{k}=\Delta T / q$, where $\Delta T$ denotes the temperature drop across the interface and $q$ the net heat flux flowing across the contact area.

We examine $R_{k}$ through nonequilibrium molecular dynamics (MD) simulations ${ }^{12}$ for a Silicon (Si)-Germanium (Ge) superlattice that is illustrative of semiconductor applications. ${ }^{13,14}$ Figure 1 presents a representative temperature distribution along the $x$-wise direction of a $20.0 \mathrm{~nm}(x) \times 2.5 \mathrm{~nm}$ $(y) \times 2.5 \mathrm{~nm}(z)$ cuboid domain. It includes a $10.0 \mathrm{~nm} \times 2.5$ $\mathrm{nm} \times 2.5 \mathrm{~nm}$ solid Si lattice extending from $0-10 \mathrm{~nm}$ along the $x$-wise direction and a $10.0 \mathrm{~nm} \times 2.5 \mathrm{~nm} \times 2.5 \mathrm{~nm}$ Ge lattice from 10-20 $\mathrm{nm}$. We neglect lattice mismatch during the epitaxial growth of Si films on Ge, or vice versa, which can influence interfacial thermal transport ${ }^{15,16}$ and consider idealized defect-free Si-Ge interfaces that have smooth contacts.

The MD simulations are conducted using the LAMMPS code. $^{17}$ The Si-Si, Ge-Ge, and $\mathrm{Si}-\mathrm{Ge}$ interactions are described through the Tersoff potential ${ }^{18,19}$ which is based on the concept of bond order, i.e., the bond between the atoms $i$ and $j$ is weakened by the presence of other bonds involving atom $i$. Periodic boundary conditions are employed in the $y$-and $z$-directions for the MD simulations while free

\footnotetext{
${ }^{\text {a) }}$ Author to whom correspondence should be addressed. Electronic mail: ikpuri@vt.edu. Tel.: +1-540-231-3243. Fax:+1-540-231-4574.
}

surface conditions are imposed along the $x$-wise direction. The system is initialized at $300 \mathrm{~K}$ and equilibrates with a Langevin thermostat with a coupling time of $0.1 \mathrm{ps}$ for $2 \mathrm{~ns}$.

A temperature gradient is imposed on the system by heating the $\mathrm{Si}$ atoms between $0-0.5 \mathrm{~nm}$ at $330 \mathrm{~K}$ and cooling the Ge atoms between $19.5-20.0 \mathrm{~nm}$ at $270 \mathrm{~K}$. The temperatures are maintained constant by rescaling the atomic velocities every timestep. ${ }^{20}$ The entire system is thereafter allowed to behave freely with constant volume and energy over the next 11 ns. All simulations used a timestep of 0.002 ps.

The simulated temperatures are recorded every $20 \mathrm{ps}$ and averaged over the last $1 \mathrm{~ns}$ in successive $0.3 \mathrm{~nm} \times 2.5$ $\mathrm{nm} \times 2.5 \mathrm{~nm}$ slabs. The resulting spatial temperature distribution is presented in Fig. 1. The interfacial thermal resistance at $\sim 10 \mathrm{~nm}$ produces a temperature discontinuity of $\sim 5 \mathrm{~K}$ across the Si-Ge interface. ${ }^{21}$ Higher temperatures enhance lattice vibrations since the local atoms also possess larger kinetic energies. The vibrations in the Si lattice propagate, transferring energy to cooler regions in the superlattice and also scattering internally. The difference in the phonon propagation speeds across the $\mathrm{Si}-\mathrm{Ge}$ interface, leads to a mismatch in phonon frequencies. Thus, phonons travelling through Si scatter at the

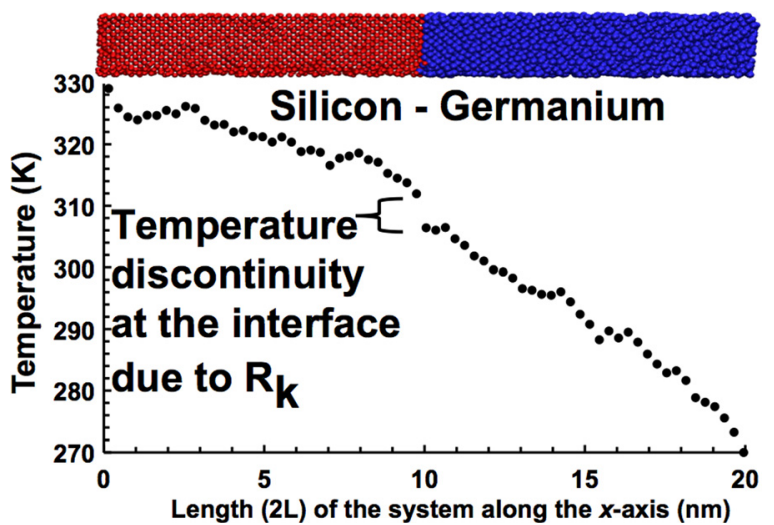

FIG. 1. (Color online) Spatial temperature distribution in a Si-Ge superlattice. Thermal transport is induced by hot $\mathrm{Si}$ atoms that lie between $x=0$ and $0.5 \mathrm{~nm}\left(T_{S i}=330 \mathrm{~K}\right)$ that transfer heat to cooler Ge atoms between $x=19.5$ and $20.0 \mathrm{~nm}\left(T_{G e}=270 \mathrm{~K}\right)$. A temperature discontinuity of $\sim 5 \mathrm{~K}$ is observed at the interface at $x=10 \mathrm{~nm}$ due to the interfacial thermal resistance $R_{k}$. 
interface, only partially transferring their energy to Ge. This impedance to heat conduction results in the temperature discontinuity at the interface. Overall, the bulk system follows the empirical Fourier's law of continuum heat conduction ${ }^{4}$ in regions further removed from its boundaries.

Since the heat transfer changes from ballistic transport at length scales smaller than the characteristic phonon mean free path to diffusive transport for larger systems, ${ }^{1}$ the material thermal conductivity, a required parameter in continuum relations, also changes. ${ }^{2,22}$ We use equilibrium MD to simulate $\mathrm{Ge}$ cubes of various edge dimensions $L$ to describe the dependence of its thermal conductivity $k_{G e}$ on length. ${ }^{23-25}$

Thermal transport across the length scales is next coupled into a modified continuum simulation using transport coefficients that depend on the dimensions of the superlattice. Instead of interpreting the interfacial temperature difference as a jump or a discontinuity, we model the interface as a material with a very small thermal conductivity (and correspondingly large resistance). This requires an accurate characterization of the effects of system size on $R_{k}$. We employ nonequilibrium MD to simulate various forms of the Si-Ge superlattice described through Fig. 1 by altering the $x$-wise length of $\operatorname{Si} L_{x-S i}$ between 1 and $100 \mathrm{~nm}$. In all these simulations, $L_{x-S i}=L_{x-G e}$. The net heat flux through the interface is calculated by time averaging the energy supplied to the warmer $\mathrm{Si}$ and removed from the cooler Ge atoms to maintain their temperatures at constant values. ${ }^{21}$

Figure 2(a) presents the change in $R_{k}$ with respect to the system length. The simulated $R_{k}$ values agree with the literature. ${ }^{21}$ With increasing $L_{x-S i}, R_{k}$ decreases, which follows from our argument about the length dependence of thermal conductivity. The reduction in $R_{k}$ is relatively large between 1 and $40 \mathrm{~nm}$, which are lengths smaller than the mean free paths of phonons. ${ }^{1}$ For larger dimensions, the resistance approaches a constant value. Likewise, the temperature drop at the interface is larger $(>10 \mathrm{~K})$ when $L_{x-S i}<20 \mathrm{~nm}$ but gradually diminishes to a constant value $(3-5 \mathrm{~K})$ as the system size increases. Overall, the relation $R_{k} \alpha L^{-\beta}$ holds.

When the boundary temperatures are maintained constant in the simulations and the system length is varied, the net heat flux through the system changes, which also influences $R_{k}{ }^{26,27}$ Therefore, we again simulate the same set of samples with varying lengths, but instead of maintaining constant boundary temperatures, we supply and extract the same energy $\left(=8 \times 10^{-8} \mathrm{~W}\right)$ to the hot and cold boundaries during each timestep. This ensures that heat fluxes through the different systems are identical. Figure 2(b) presents the variation in $R_{k}$ with $L_{x-S i}$, which is overall similar to the predictions in Fig. 2(a). Both $R_{k}$ and $\Delta T$ decrease by larger amounts for $L_{x-S i}<30 \mathrm{~nm}$ but then assume constant values. This again demonstrates the presence of a characteristic interfacial resistance even at bulk scales, which follows the $R_{k} \propto L^{-\beta}$ relationship. Also, we find that $R_{k}$ is dependent only on the dimensions along the direction of heat conduction, not on those perpendicular to it. $^{23}$

A schematic for the proposed technique is in Fig. 3 where the interface is considered as another material contained between the $\mathrm{Si}-\mathrm{Ge}$ superlattice. To demonstrate its applicability, we select the $20 \mathrm{~nm}(x) \times 2.5 \mathrm{~nm}(y) \times 2.5 \mathrm{~nm}$ $(z)$ cuboid simulation domain in the configuration of Fig. 1.

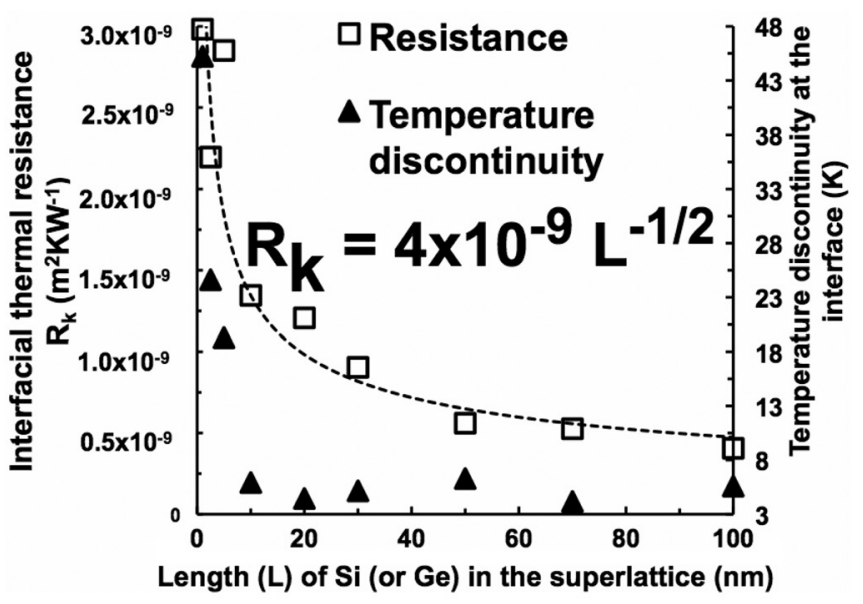

(a)

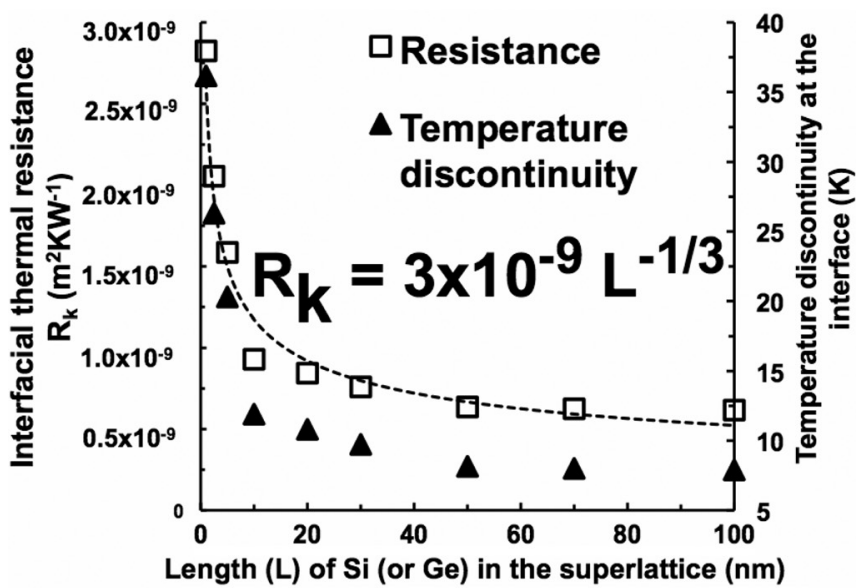

(b)

FIG. 2. Variation of $R_{k}$ and interfacial temperature discontinuity $\Delta T$ for different lengths of the superlattice obtained from the MD simulations when (a) the temperatures at the boundaries are constant, i.e., $T_{S i}=330 \mathrm{~K}$ and $T_{G e}=270 \mathrm{~K}$, and (b) a constant energy of $8 \times 10^{-8} \mathrm{~W}$ is supplied and simultaneously extracted during every timestep from the superlattice boundaries. The reduction in $R_{k}$ with increasing $L$ follows a similar relation for both cases. The decrease is relatively large for system dimensions smaller than the mean free path of phonons, ${ }^{1}$ but $R_{k}$ assumes a constant value at larger lengths. The temperature discontinuities follow a similar trend. At length scales larger than the phonon mean free path, $\Delta T$ has a constant material specific characteristic value.

Since interfacial effects influence $\sim 2$ atomic layers on either side of the contact location, ${ }^{6}$ the dimensions of the interfacial material are assumed to be $1.2 \mathrm{~nm}(x) \times 2.5 \mathrm{~nm}(y) \times 2.5 \mathrm{~nm}$ (z) that lies between 9.4 and $10.6 \mathrm{~nm}$ along the $x$-wise direction.

The Fourier law for steady-state heat conduction suggests that the overall heat flux across a system of composite slabs with different thermal conductivities is $q=\left(T_{H O T}\right.$ $\left.-T_{C O L D}\right) / R_{\text {TOTAL. }}{ }^{4}$ Here, the total thermal resistance $R_{\text {TOTAL }}$ $=L_{x-S i} / k_{S i}+R_{k}+L_{x-G e} / k_{G e}$ accounts for resistances due to the length-dependent thermal conductivities of $\mathrm{Si}$ and $\mathrm{Ge}$ as well at the Kapitza resistance of the assumed interfacial material. ${ }^{4,21,28}$ Again, the flux $q=\left(T_{H O T}-T_{i-S i}\right) /\left(L_{x-S i} / k_{S i}\right)$ $=\left(T_{i-S i}-T_{i-G e}\right) / R_{k}=\left(T_{i-G e}-T_{C O L D}\right) /\left(L_{x-G e} / k_{G e}\right)$ leads to a temperature distribution within each material. $T_{i-S i}$ and $T_{i-G e}$ are the temperatures at the boundaries of the interfacial material in contact with $\mathrm{Si}$ and $\mathrm{Ge}$, respectively. Using $T_{H O T}=330 \mathrm{~K}, T_{C O L D}=270 \mathrm{~K}$, the length-dependent $k_{S i}$ 
Silicon - Interface - Germanium

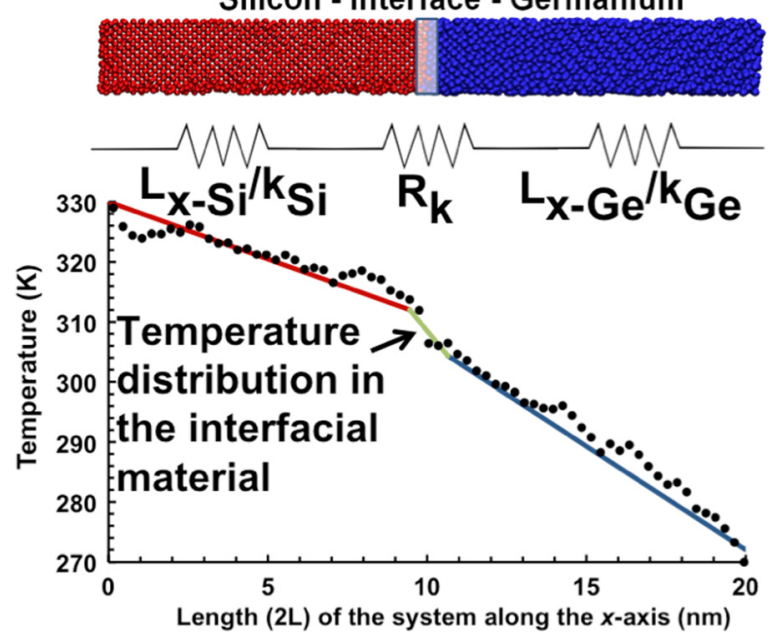

FIG. 3. (Color online) Spatial temperature distribution shown through continuous straight lines for a $20 \mathrm{~nm}$ ( $x$-wise) long Si-Ge superlattice obtained using a modified continuum approach that considers the interface as a distinct material with a thermal resistance $R_{k}$. The thermal transport is induced by hot $\mathrm{Si}$ atoms that lie between $x=0$ and $0.5 \mathrm{~nm}\left(T_{S i}=330 \mathrm{~K}\right)$ that transfer heat to cooler Ge atoms between $x=19.5$ and $20.0 \mathrm{~nm}\left(T_{G e}=270 \mathrm{~K}\right)$. The resistances provided by the Si lattice $\left(L_{x-S i} / k_{S i}\right)$, interface $\left(R_{k}\right)$, and $\mathrm{Ge}$ $\left(L_{x-G e} / k_{G e}\right)$ that contribute to the overall heat transfer are shown. The filled circles represent the MD simulation results from Fig. 1 for comparison.

$=5.38 \mathrm{Wm}^{-1} \mathrm{~K}^{-1},{ }^{22} k_{G e}=3.33 \mathrm{Wm}^{-1} \mathrm{~K}^{-1}$, and $R_{k}=1.26$ $\times 10^{-9} \mathrm{Wm}^{-1} \mathrm{~K}^{-1}$ obtained from the simulations lead to the temperature distribution presented in Fig. 3. Not only does it reproduce the expected continuum behavior away from the interface, but this also replicates the temperature discontinuity across the interface that is predicted using $\mathrm{MD}$, which is superimposed to validate our technique.

In summary, thermal transport changes from a ballistic form into diffusive behavior as the length increases from the nano- to the mesoscale. An accurate representation of heat transfer across solid-solid interfaces should account for the corresponding changes in both the bulk and the interfacial thermal resistance with varying length. We provide a modified continuum approach that incorporates the interfacial temperature discontinuity from atomistic simulations of dissimilar solids. The technique treats the interface as another material with a resistance $R_{k}$ that depends on the overall system length. As the system sizes increases, lattice vibrations that carry thermal energy scatter inside the bulk material and energy losses at the interface are reduced. This increases the material thermal conductivities simultaneously decreasing the interfacial thermal resistance according to $R_{k} \alpha L^{-\beta}$. The contact area does not exert an appreciable influence on ther- mal transport. The Fourier heat conduction relation is modified to account for $R_{k}$. For our Si-Ge model system, the approach is able to accurately replicate the effects of $R_{k}$ and predict the temperature discontinuity predicted by MD simulations alone. A future comparison of the phonon density of states of the atoms in the bulk material and at the interface for different superlattice dimensions should further clarify the role of the thermal resistance.

We thank Tim Tomlin of the Virginia Tech Department of Engineering Science and Mechanics for assisting us with the use of the LCC cluster.

${ }^{1}$ G. Chen, Int. J. Therm. Sci. 39(4), 471 (2000).

${ }^{2}$ D. P. Sellan, E. S. Landry, J. E. Turney, A. J. H. McGaughey, and C. H. Amon, Phys. Rev.B 81(21), 214305 (2010).

${ }^{3}$ P. K. Schelling, S. R. Phillpot, and P. Keblinski, Phys. Rev. B 65(14), 144306 (2002).

${ }^{4}$ F. P. Incropera, Fundamentals of Heat and Mass Transfer (Wiley, Hoboken, NJ, 2007).

${ }^{5}$ M. Hu, J. V. Goicochea, B. Michel, and D. Poulikakos, Appl. Phys. Lett. 95(15), 51903 (2009).

${ }^{6}$ S. Murad and I. K. Puri, Chem. Phys. Lett. 467(1-3), 110 (2008).

${ }^{7}$ R. J. Stevens, L. V. Zhigilei, and P. M. Norris, Int. J. Heat Mass Trans. 50(19-20), 3977 (2007).

${ }^{8}$ L. J. Challis, J. Phys. C 7(3), 481 (1974).

${ }^{9}$ G. L. Pollack, Rev. Mod. Phys. 41(1), 48 (1969).

${ }^{10}$ E. T. Swartz and R. O. Pohl, Rev. Mod. Phys. 61(3), 605 (1989).

${ }^{11}$ S. Murad and I. K. Puri, Appl. Phys. Lett. 95(5), 051907 (2009).

${ }^{12}$ J. M. Haile, Molecular Dynamics Simulation Elementary Methods (Wiley, New York, USA, 1997).

${ }^{13}$ B. Becker, P. K. Schelling, and S. R. Phillpot, J. Appl. Phys. 99(12), 114301 (2006).

${ }^{14}$ S. Volz, J. B. Saulnier, G. Chen, and P. Beauchamp, Microelectron. J. 31(9-10), 815 (2000).

${ }^{15}$ P. Y. Hsiao, Z. H. Tsai, J. H. Huang, and G. P. Yu, Phys. Rev. B 79(15), 155414 (2009).

${ }^{16}$ M. Hu, J. V. Goicochea, B. Michel, and D. Poulikakos, Nano Lett. 10(1), 279 (2010).

${ }^{17}$ S. Plimpton, J. Comput. Phys. 117(1), 1 (1995).

${ }^{18}$ J. Tersoff, Phys. Rev. B 37(12), 6991 (1988).

${ }^{19}$ J. Tersoff, Phys. Rev. B 39(8), 5566 (1989).

${ }^{20}$ G. Balasubramanian, S. Banerjee, and I. K. Puri, J. Appl. Phys. 104(6), 064306 (2008).

${ }^{21}$ V. Samvedi and V. Tomar, Nanotechnology 20(36), 365701 (2009).

${ }^{22}$ Y. W. Yang, X. J. Liu, and J. P. Yang, Mol. Simul. 34(1), 51 (2008).

${ }^{23}$ See supplementary material at http://dx.doi.org/10.1063/1.3607477 for Ge thermal conductivity dependence on system dimensions, and the thermal resistance dependence on the interfacial contact area between the $\mathrm{Si}-\mathrm{Ge}$ superlattice.

${ }^{24}$ A. Guajardo-Cuellar, D. B. Go, and M. Sen, J. Chem. Phys. 132(10), $104111(2010)$.

${ }^{25}$ J. J. Dong, O. F. Sankey, and C. W. Myles, Phys. Rev. Lett. 86(11), 2361 (2001).

${ }^{26}$ S. Murad and I. K. Puri, Appl. Phys. Lett. 92, 133105 (2008).

${ }^{27}$ P. L. Kapitza, J. Phys. USSR 4, 181 (1941).

${ }^{28}$ S. Murad and I. K. Puri, Chem. Phys. Lett. 476(4-6), 267 (2009). 